
\title{
25 Research Soure \\ Two Weeks High Glucose is Enough to Induce Liver and Gut Lesion
}

\section{Cunyun Min ( $\nabla$ mincunyun@gdph.org.cn )}

Guangdong Provincial People,s Hospital,Guangdong Academy of Geriatrics

\section{Tingting Fu}

Guangdong Provincial People,s Hospital,Guangdong Academy of Geriatrics

\section{Yu Du}

Guangdong Provincial People,s Hospital,Guangdong Academy of Geriatrics

\section{Wei Tan}

Guangdong Provincial People,s Hospital,Guangdong Academy of Geriatrics

\section{Xiuhui Huang}

Guangdong Provincial People,s Hospital,Guangdong Academy of Geriatrics

\section{Feng Zhan}

Guangdong Provincial People,s Hospital,Guangdong Academy of Geriatrics

\section{Research}

Keywords: high glucose infusion, oral high glucose, liver, intestinal mucosa, gut microbiota

Posted Date: July 12th, 2021

DOI: https://doi.org/10.21203/rs.3.rs-684386/v1

License: (c) (1) This work is licensed under a Creative Commons Attribution 4.0 International License. Read Full License 


\section{Abstract}

Background: High glucose is critical for diabetes.But in which way it induces diabetes, and which organ trigger the formation of diabetes are not clear.This study is to evaluate the effect of shot time high glucose on different organs,to make clear this question.

Methods: Twelve weeks old SD rats were randomly assigned to control group,high glucose infusion (HGI) group and oral high glucose (OHG) group.Fasten blood sugar,TNF-a and IL-6 were measured.Kidneys,intestine and liver samples were collected for pathological examination.Feces of rats were collected for gut microbiota tests.

Results: The results indicated that short time high glucose induced hyperglycemia lasted for at least 2 weeks after ceasing of high glucose.It increased serum levels of IL-6 and TNF-a obviously.It led to small intestinal mucosa injury, obvious steatosis of hepatocytes, and broke the balance of gut microbiota.OHG led to swelling and necrosis of individual intestinal villi.HGI led to necrosis and disappearence of cells in the upper layer of intestinal mucosa. The lesion was confined to the mucosa. There is not obvious biopsy change in kidney and pancreas.

Conclusions: Short time high glucose induced lesion of liver and intestine,broken the balance of gut microbiota.All of these led to inflammation and triggered diabetes.

\section{Introduction}

Type 2 Diabetes mellitus(T2DM) is rapidly increasing worldwide. It is linked to higher medical costs[1, 2]. Diabetes treatments have made some success.But their effect is not always sustained and their use may associated with undesirable side effects, such as hypoglycaemia. Insufficient blood glucose control led to complications and early mortality.The complications of diabetes, like cardiovascular disease, nephropathy, neuropathy, liver damage and so on happen quickly after formation of T2DM[3].Some clinical experiments confirmed the "legacy effect" or "metabolic memory" of prior glycemic control.Longterm cardiovascular benefits of good glycemic control early in the course of diabetes has received more and more attention[4, 5].More and more evidence suggest that many patients with type 2 diabetes can follow a nonalbuminuric pathway to renal function loss, even after accounting for the use of renoprotective agents[6, 7].It is important to make clear the relationship between high glucose intake and formation of diabetes and stop the progression of prediabetes into diabetes.

Hyperglycemia is critical in the genesis of diabetic complications. Poor glycemic control is an independent predictor of the development and progression of complications. The tight control of glucose concentration is determined by a balance between glucose absorption from the intestine, glucose production by the liver, and glucose uptake from the plasma.

The liver plays a major role in the regulation of glucose metabolism. The relationship between T2DM and chronic liver diseases (CLD) was discussed actively in recent years.Patients with diabetes have an 
increased risk of developing CLD.Steatosis, portal fibrosis, and diabetic steatonecrosis are frequent patterns of hepatic involvement that take place during diabetes[8]. Patients withT2DM are prone to develop nonalcoholic fatty liver disease (NAFLD), and NAFLD itself is associated with a doubled risk of incident T2DM.Many patients with CLD have abnormal glucose metabolism ultimately leads to impaired glucose tolerance and the development of diabetes. The pathogenesis of impaired glucose metabolism during chronic liver disease has not yet been fully understood. The potential of targeting the liver to normalize blood glucose levels has not been fully exploited[9-11]. The molecular mechanisms controlling hepatic gluconeogenesis and glycogen storage are not very clear.Further clinical and experimental studies should clarify this issue.We were to make clear how long will it take for high glucose to induce liver lesion.

Intestinal mucosa is the position of oral glucose get into blood. The gut microbiota is a large and complex microbial ecosystem that maintains the homeostasis of the body and environment. The microbiota consumes carbohydrates that are not easily digested by the body.Human gut microbiome is a promising target for managing T2DM.Short-chain fatty acids (SCFAs) are produced by the fermentation of dietary fiber by the gut microbiota and are beneficial to the health of the body. Insufficient SCFAs productions are associated with T2DM[12, 13]. This indicated that Intestinal mucosa and gut microbiota play an important role in the glycemic control.But the change of Intestinal mucosa and gut microbiota after a short time of high glucose intake is not clear.

Sugar consumption is regarded as a major risk for the development of obesity.Diets enriched in sugars including the intake of sugar-sweetened beverages have been consistently linked to the increased risk of obesity, T2DM, and cardiovascular disease. Dietary glucose increased serum glucose and insulin concentrations in the postprandial state.Studies in experimental animals and in man have demonstrated that chronic elevation in the plasma glucose concentration impairs insulin action.Chronic hyperglycemia causes insulin resistance, but the short time glucotoxicity and the underlying mechanisms are unclear[14, 15].

High glucose is a critical factor for formation and progression of diabetes. But in which way it induces diabetes, and which organ triggers diabetes are not very clear till now.Most studies are restricted to effects of sugars only on oral high glucose or high glucose infusion on only one or two organs. Therefore, we aim to evaluate the different influence of short time oral high glucose or high glucose infusion sugars on different organs at the same. We fed or infused liquid high glucose, and analyzed their influence on liver,pancreas, kidneys,intestine as well as the development gut microbial dysbiosis at same time.

\section{Materials And Methods}

\section{Experimental Animals}

This study was performed with 30 male specifific pathogen-free (SPF)-grade Sprague-Dawley rats, aged 12 weeks and weighing approximately $200 \pm 20 \mathrm{~g}$. 
Rats were purchased from Experiment Animal Center of Guangdong Province. (approval No: SCXK (Yue)-2013-0002, Guangdong, China). All rats were raised under a light-controlled condition (12 h

light/12 $\mathrm{h}$ dark) and in a temperature-controlled $(23 \pm 2 \circ \mathrm{C})$ room with food and water available.

All animal experiments were conducted in accordance with the committee guidelines of the Guangdong Provincial People's Hospital and approved by the Institutional Animal Care and Use Committee of Guangdong Provincial People's Hospital, No.KY-D-2019-082-01.

\section{Experimental Design}

After adaptive feeding, all rats were randomly assigned into three groups, normal diet,ND, $n=10$; oral high glucose intake, $\mathrm{OHG}, \mathrm{n}=10$; high glucose infusion, $\mathrm{HG}, \mathrm{n}=10$. The $\mathrm{OHG}$ group rats were fed with $50 \%$ high glucose at a dose of $2.5 \mathrm{~g} / \mathrm{kg} /$ day for 2 weeks. The IHG group rats were treated with $50 \%$ high glucose by infusion at a dose of $2 \mathrm{~g} / \mathrm{kg} /$ day for 2 weeks. The normal diet group received an equivalent amount of saline for the same period. During the experiment, all rats was received the same standard chow. Fasting blood sugar level (FBS) was measured by a glucometer (Abbot, Alameda, CA, USA) every week in all animals. After 4 weeks of treatment, all rats were sacrificed; $10 \%$ chloral hydrate (3 mL/kg i.p., Sigma, St. Louis, MO, USA) was used to reduce pain. The following samples were collected from the rats:Feces were collected individually for at least 3 days for each animal.Blood samples were collected by the retro-orbital plexus for biochemical assays.Kidneys, intestine, pancreas, and liver samples were also collected.

\section{Detection of cytokines and chemokines}

In order to evaluate the level of inflammation in rats, ELISA was used to measure serum levels of proinflammatory cytokines interleukin(IL)- 6 and tumor necrosis factor (TNF)-a according to the anufacturer's instructions (ELISA, Nanjing Jiancheng Bioengineering Institute, Nanjing, China). Twenty-four-hour microalbuminuria was also assessed by ELISA, according to the manufacturer's instructions.

\section{Histopathological examinations}

The small intestine was removed and divided longitudinally, washed and placed in complete media.Sections of intestine, liver, and epididymal white adipose were fixed in $10 \%$ formalin, paraffin embedded, and stained with hematoxylin and eosin. Oil Red 0 staining was performed on frozen sections fixed in $4 \%$ paraformaldehyde and sucrose protected. For comparison of histologic differences, three sections were blindly selected per sample from each mouse and quantified using ImageJ software.

\section{Gut Microbiota Analysis}

\section{DNA extraction and PCR amplification}

Microbial DNA was extracted using the HiPure Soil DNA Kits (or HiPure Stool DNA Kits) (Magen, Guangzhou, China) according to manufacturer's protocols. The 16S rDNA V4 region of the ribosomal RNA 
gene were amplified by PCR $₫ 95^{\circ} \mathrm{C}$ for 2 min, followed by 27 cycles at $98^{\circ} \mathrm{C}$ for $10 \mathrm{~s}, 62^{\circ} \mathrm{C}$ for $30 \mathrm{~s}$, and $68^{\circ} \mathrm{C}$ for $30 \mathrm{~s}$ and a final extension at $68^{\circ} \mathrm{C}$ for $10 \mathrm{~min} \rrbracket$ using primers

Arch519F『CAGCMGCCGCGGTAA\Arch915R囚GTGCTCCCCCGCCAATTCCT, where the barcode is an eightbase sequence unique to each sample. PCR reactions were performed in triplicate

$50 \mu \mathrm{L}$ mixture containing $5 \mu \mathrm{L}$ of $10 \times$ KOD Buffer, $5 \mu \mathrm{L}$ of $2.5 \mathrm{mM}$ dNTPs, $1.5 \mu \mathrm{L}$ of each primer $(5 \mu \mathrm{M}), 1$ $\mu \mathrm{L}$ of KOD Polymerase, and $100 \mathrm{ng}$ of template DNA.Amplicons were extracted from $2 \%$ agarose gels and purified using the AxyPrep DNA Gel Extraction Kit (Axygen Biosciences, Union City, CA, U.S.) according to the manufacturer's instructions and quantified using ABI StepOnePlus Real-Time PCR System (Life Technologies,Foster City, USA). Purified amplicons were pooled in equimolar and paired-end sequenced (2 $x$

250) on an Illumina platform according to the standard protocols. The raw reads were deposited into the NCBI Sequence Read Archive (SRA) database (Accession Number: SRP ${ }^{\star \star \star \star \star \star *) . ~}$

\section{Bioinformatics analysis}

Raw data were further filtered according to the following rules using FASTP (https://github.com/OpenGene/fastp):(1) Removing reads containing more than $10 \%$ of unknown

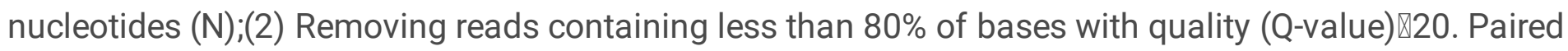
end clean reads were merged as raw tags using FLSAH(version 1.2.11) with a minimum overlap of $10 \mathrm{bp}$ and mismatch error rates of $2 \%$. Noisy sequences of raw tags were filtered by QIIME (version 1.9.1) pipeline under specific filtering conditions to obtain the high-quality clean tags. Clean tags were searched against the reference database (http://drive5.com/uchime/uchime_download.html) to perform referencebased chimera checking using UCHIME algorithm

(http://www.drive5.com/usearch/manual/uchime_algo.html). All chimeric tags were removed and finally obtained effective tags were used for further analysis. The effective tags were clustered into operational taxonomic units (OTUs) of $\geq 97 \%$ similarity using UPARSE pipeline. The tag sequence with highest abundance was selected as representative sequence within each cluster. Between groups Venn analysis was performed in $\mathrm{R}$ project (version

3.4.1) to identify unique and common OTUs. The representative sequences were classified into organisms by a naive Bayesian model using RDP classifier(version 2.2) based on SILVA Database (https://www.arb-silva.de/), with the confidence threshold values ranged from 0.8 to 1 . The abundance statistics of each taxonomy were visualized using Krona (version 2.6). Biomarker features in each group were screened by Metastats (version 20090414) and LEfSe software[9] (version 1.0).Chao1, Simpson and all other alpha diversity index were calculated in QIIME. OTU rarefaction curve and rank abundance curves were plotted in QIIME. Alpha index comparison between groups was calculated by Welch's t-test and Wilcoxon rank test in R project. Alpha index comparison among groups was computed by Tukey's HSD test and Kruskal-Wallis $\mathrm{H}$ test in $\mathrm{R}$ project. Weighted and unweighted unifrac distance matrix were generated by QIIME. Multivariate statistical techniques including PCA (principal component analysis), 
PCoA (principal coordinates analysis) and NMDS (non-metric multi-dimensional scaling) of (Un) weighted unifrac distances were calculated and plotted in R project. Statistic analysis of Welch's t-test, Wilcoxon rank test, Tukey's HSD test, Kruskal-Wallis H test, Adonis (also called Permanova) and Anosim test was calculated using R project. The KEGG pathway analysis of the OTUs was inferred using Tax4Fun(version 1.0).

\section{Results}

\section{Short time high glucose led to persistent hyperglycemia}

In order to compare the influence of high glucose infusion and oral high glucose on blood glucose.Fasten blood sugar level (FBS) was measured before glucose taking,14days after glucose taking,7 days and 14 days after ceasing of glucose taking.Both oral high glucose and high glucose infusion increased blood glucose obviously.The blood glucose decreased slowly 7 days after ceasing of glucose taking in both groups. The rats blood glucose in both groups was higher than normal diet rats till 14 days after ceasing of high glucose.There is no difference between oral high glucose intake and high glucose infusion. See figure 1.

\section{Short time high glucose induced inflammation}

At the end of high glucose intake,serum levels of inflammatory cytokines IL- 6 and TNF-a were measured to evaluate the level of inflammation in rats. Oral high glucose and high glucose infusion increased serum levels of IL- 6 and TNF-a obviously. High glucose infusion was a little more intensive than oral high glucose. See table 1 .

\section{Short time high glucose increased hepatic lipid accumulation}

Hepatic fat accumulation was investigated 14 days after ceasing of high glucose. Both oral high glucose and high glucose infusion induced obvious steatosis in rats liver.A large number of hepatocytes were injured. There is no difference between oral high glucose intake and high glucose infusion.See figure 2.

\section{Short time high glucose induced damage of intestinal mucosa}

Small intestinal mucosa of rats were collected for pathological tests.Both oral high glucose and high glucose infusion induced the small intestinal mucosa injury. Oral high glucose led to swelling and necrosis of individual intestinal villi.Some epithelial cells disappeared after 2 weeks of oral high glucose.High glucose infusion led to necrosis and disappearence of cells in the upper layer of intestinal mucosa. The lesion was confined to the mucosa.See figure 3.

\section{Changes of gut microbiota}

To elucidate the mechanism of the effect of high glucose, we investigated the impact of high glucose on the gut microbiota in SD rats. 16 S rDNA sequencing was used to assess changes in the fecal microbiota 
of SD rats with oral high glucose or high glucose infusion.Akkermansiaceae $\mathbb{Z}$ Lactobacillaceae $\mathbb{}$ Muribaculaceae\Ruminococcaceae『Peptostreptococcacea\and Clostridiaceae_ 1 account for $60 \%$ of gut microbiota in SD rat.Rats in two groups lost gut microbial diversity.It was characterized by a lower proportion of Akkermansiaceae and a markedly increased proportion of Lactobacillaceae,Muribaculaceae, Ruminococcaceae,Peptostreptococcacea.

See figure 4,figure 5 and figure6.

\section{Discussion}

Sugar consumption is regarded as a major risk for the development of obesity and diabetes.Prediabetes or intermediate hyperglycemia is a high risk state for developing T2DM[16,17]. We compared the effect of oral high glucose and high glucose infusion on blood glucose.Both of them increased blood glucose obviously.And they led to persistent hyperglycemia till two weeks later. They both delayed the weight gain of rats. These results confirmed that sugar is a critical cause of diabetes. We need to reduce the intake of sugar.

Inflammation involves a tightly regulated process, divided into two complementary subsystems: the innate immune system and the highly adaptive immune system. More and more study indicated that diabetes is a kind inflammation. It is now accepted that the obesity-associated chronic, low-grade systemic inflammation is a major underlying factor for the development of many metabolic diseases[18].Our results indicated that only 2 weeks of oral high glucose or high glucose infusion induced inflammation in rats.

Clinical and pathophysiological studies have shown type 2 diabetes to be a condition mainly caused by excess fat accumulation in the liver and pancreas. Excess fat worsens hepatic responsiveness to insulin, leading to increased glucose production. Removal of excess fat from liver via substantial weight loss can normalise hepatic insulin responsiveness[19,20]. Negative energy balance in type 2 diabetes causes a profound fall in liver fat content resulting in normalisation of hepatic insulin sensitivity within 7 days.As the period of negative energy balance extends and liver fat levels fall to low normal, the rate of export of triacylglycerol from the liver falls. The primary care-based Diabetes Remission Clinical Trial showed that $46 \%$ of people with type 2 diabetes could achieve remission at 12 months, and $36 \%$ at 24 months, mediated by weight loss.Glucotoxicity and lipotoxicity are key features of type 2 diabetes mellitus, but their molecular nature during the early stages of the disease remains to be elucidated[21,22]. Our study indicated that both oral high glucose and high glucose infusion induced obvious steatosis in rats liver in just 2 weeks.But there was not obvious lesion on kidneys and pancreas.Liver may be the first organ damaged by high glucose.Is liver the trigger of diabetes? We need further study.

Intestinal epithelium is characterized by its remarkable self-renewal ability. The crypt base columnar cells marked by Lgr 5 represent the actively proliferating stem cells that mediate the daily renewal of intestinal epithelium. The constant renewal cycle takes place in a hostile environment characterised by the presence of bacterial toxins and metabolites,dietary antigens and mutagens, and immunological cytokines and 
oxidative stress. The intestine has been implicated as a key organ that critically contributes to the development of obesity-associated chronic inflammation and systemic insulin resistance, and metabolic dysregulation[23,24].Glucose directly stimulates intestinal epithelial cells[25].Our results indicated that both oral high glucose and high glucose infusion led to the small intestinal mucosa injury.The damage from oral high glucose was more serious than high glucose infusion.It influenced the villi and mucosa at the same time. This may be the result of direct contaction.

The gastrointestinal (GI) tract is a highly complex organ composed of the intestinal epithelium layer, intestinal microbiota, and local immune system.Gut microbiota is the most diverse communities. It is in constant interaction with our body's cells and systems. Different sections of the GI tract contain distinct proportions of the intestinal microbiota[26-28]. Gut microbiota and its metabolites play pivotal roles in host physiology and pathology.Diet is one of the various factors that influences the microbiota. Intestinal microbiota modulate metabolism and associate closely with epithelial cells in the intestine[29,30].The intestinal microbiota converts ingested nutrients into metabolites that target either the intestinal microbiota population or host cells.As metabolite of intestinal microbiota, Short Chain Fatty Acids(SCFAs) are a major energy source for intestinal epithelial cells in the colon and reinforce the intestinal barrier function via multiple mechanisms.SCFAs can reduce inflammation and protect kidney.But lipopolysaccharide (LPS) comes from microbiota induces inflammation and injury of kidney,heart,cerebrovascular and other organs.A vast number of studies have demonstrated a remarkable role for the gut microbiota and their metabolites in the pathogenesis of T2DM.Accumulating evidences suggest that SCFAs regulate inflammation, energy metabolism, and blood pressure, which affects kidney function through the gut-kidney axis[31-33].Our study indicated that rats treated with high glucose lost gut microbial diversity in two weeks. It was characterized by a lower proportion of Akkermansiaceae and a markedly increased proportion of actobacillaceae, Muribaculaceae,Ruminococcaceae, Peptostreptococcacea.Decreasing of Akkermansiaceae is closely related with fat and diabetes.

\section{Conclusion}

We may conclude that high glucose induce lesion of liver and intestinal epithelium, and gut microbial dysbiosis.All these induce inflammation and trigger diabetes.

\section{Declarations}

Ethics approval and consent to participate

Written informed consents were obtained from all participants and this study

was permitted by the Ethics Committee of Guangdong Provincial People's Hospital.

\section{Acknowledgements}

No. 


\section{Author contributions}

Cunyun Min. conceived the ideas and designed the study;Tingting Fu,Yu Du, Wei Tan. collected and analysed the data;Xuhui Huang,Feng Zhan. led the writing of the manuscript. All authors contributed critically to the drafts and gave final approval for publication.

\section{Funding}

This work is supported by grants from Guangdong Provincial Natural Science (S2012010010783), and Guangdong Provincial bureau of traditional Chinese Medicine (20121261).

\section{Availability of data and materials}

Not applicable.

\section{Consent for publication}

Not applicable.

\section{Competing interests}

The authors declare that they have no conficts of interest.

\section{References}

1. Timothy M. Dall, Wenya Yang,Karin Gillespie, et al.The Economic Burden of Elevated Blood Glucose Levels in 2017: Diagnosed and Undiagnosed Diabetes, Gestational Diabetes Mellitus, and Prediabetes.Diabetes Care.2019;42:1661-1668

2. Catherine C. Cowie.Diabetes Diagnosis and Control:Missed Opportunities to Improve Health.Diabetes Care. 2019;42:994-1004

3. Filla LA, Edwards JL. Metabolomics in diabetic complications. Mol Biosyst. 2016;12(4):1090-105.

4. XiantongZou, XianghaiZhou, Linong Ji, et al.The characteristics of newly diagnosed adult early-onset diabetes: a population-based cross-sectional study.SCienTiFiC ReporTS 7:46534 | DOI:

10.1038/srep46534

5. Matthew C. Riddle,Hertzel C. Gerstein.The Cardiovascular Legacy of

Good Glycemic Control: Clues About Mediators From the DCCT/EDIC Study.Diabetes Care.2019;42:11591161.

6. Gaballa M, Farag MK. Predictors of Diabetic Nephropathy. Eur J Med. 2013;8:287-296.

7. Richard J. Maclsaac,Elif I. Ekinci.Progression of Diabetic Kidney 
Disease in the Absence of Albuminuria.Diabetes Care.2019;42:1842-1844

8. Rines AK, Sharabi K, Tavares CD, et al. Targeting hepatic glucose metabolism in the treatment of type 2 diabetes. Nat Rev Drug Discov. $2016 ; 15(11): 786-804$.

9. Sabine Kahl, Sofiya Gancheva, Klaus Straßburger, et al. Empagliflozin Effectively Lowers Liver Fat Content in Well-Controlled Type 2 Diabetes: A

Randomized, Double-Blind, Phase4, Placebo-Controlled Trial.Diabetes Care 2020;43:298-305

10. Chen M, Zheng H, Xu M, et al. Changes in hepatic metabolic profile during the evolution of STZinduced diabetic rats via an 1H NMR-based metabonomic investigation. Biosci Rep. 2019;39(4):BSR20181379.

11. Rai RC, Bagul PK, Banerjee SK. NLRP3 inflammasome drives inflammation in high fructose fed diabetic rat liver: Effect of resveratrol and metformin. Life Sci. 2020;15;253:117727.

12. Minzi Jua, Yaqun Liua, Mingyue Lia, et al. Baicalin improves intestinal microecology and abnormal metabolism induced by high-fat diet. European Journal of Pharmacology. 857 (2019) 172457

13. Yifei Zhang ,Yanyun Gu,Huahui Ren,et al.Gut microbiome-related effects of berberine and probiotics on type 2 diabetes (the PREMOTE study).

NATURE COMMUNICATIONS.(2020) 11:5015

14. Malik VS, Willett WC, Hu FB.Global obesity: trends, risk factors and

policy implications. Nat Rev Endocrinol .(2013)9: 13-27.

15. Shannon C, Merovci A, Xiong J, et al. Effect of Chronic Hyperglycemia on Glucose Metabolism in Subjects With Normal Glucose Tolerance. Diabetes. 2018;67(12):2507-2517.

16. Chiu THT, Pan WH, Lin MN, et al. Vegetarian diet, change in dietary patterns, and diabetes risk: a prospective study. Nutr Diabetes. $2018 ; 8(1): 12$.

17. Taylor R, Barnes AC. Translating aetiological insight into sustainable management of type 2 diabetes. Diabetologia. 2018;61(2):273-283.

18. Biscetti F, Ferraro PM, Hiatt WR, et al. Inflammatory Cytokines Associated With Failure of LowerExtremity Endovascular Revascularization (LER): A Prospective Study of a Population With Diabetes. Diabetes Care. 2019;42(10):1939-1945.

19. Kheiripour N, Karimi J, Khodadadi I, et al.Silymarin prevents lipid accumulation in the liver of rats with type 2 diabetes via sirtuin1 and SREBP-1c.J Basic Clin Physiol Pharmacol. 2018;29(3):301-308. 
20. aylor R, Al-Mrabeh A, Sattar N. Understanding the mechanisms of reversal of type 2 diabetes. Lancet Diabetes Endocrinol.2019;7(9):726-736.

21. Bril F, Portillo Sanchez P, Lomonaco R, et al.Liver Safety of Statins in Prediabetes or T2DM and Nonalcoholic Steatohepatitis: Post Hoc Analysis of a Randomized Trial. J Clin Endocrinol Metab. 2017;102(8):2950-2961.

22. Maki Tsujita, Mohammad Anwar Hossain, Rui Lu, et al.Exposure to High Glucose Concentration Decreases Cell Surface ABCA1 and HDL Biogenesis in Hepatocytes.J Atheroscler Thromb, 2017; 24: $1132-1149$

23.Sigal Leviatan,Eran Segal.Identifying gut microbes that affect human health.Nature.2020;587:373

24. Stefanie Haase,Aiden Haghikia,Nicola Wilck,et al.Impacts of microbiome metabolites on immune regulation and Autoimmunity. Immunology.2018;154:230-238

25. Rachel Botchlett,Honggui Li,XinGuo,et al.Glucose and Palmitate Differentially Regulate PFKFB3/iPFK2 and Inflammatory Responses in Mouse Intestinal Epithelial Cells.Scientific Reports.2016;6:28963

26. Leviatan S, Segal E. Identifying gut microbes that affect human health. Nature. 2020;587(7834):373374.

27. Sittipo P, Shim JW, Lee YK. Microbial Metabolites Determine Host Health and the Status of Some Diseases. Int J Mol Sci. 2019;20(21):5296.

28. Moon Ho Do, Eunjung Lee,Mi-Jin Oh,et al.High-Glucose or -Fructose Diet Cause Changes of the Gut Microbiota and Metabolic Disorders in rats without Body Weight Change.Nutrients. 2018, 10, 761

29.Tao Zhou, Yoriko Heianza,Yuhang Chen, et al.Circulating Gut Microbiota Metabolite Trimethylamine NOxide (TMAO) and Changes in Bone Density in Response to Weight Loss Diets: The POUNDS Lost Trial.Diabetes Care 2019;42:1365-1371

30. Sul A Lee,Martina Cozzi,Errol L. Bush, et al.Distant Organ Dysfunction in Acute Kidney Injury: A Review.Am J Kidney Dis. 2018; 72(6): 846-856.

31. stefanie Haase, Aiden Haghikia, Nicola Wilck, et al.Impacts of microbiome metabolites on immune regulation and autoimmunity.Immunology.2018;154, 230-238

32. Jordan Whitt, Vivienne Woo, Patrick Lee, et al.Disruption of Epithelial HDAC3 in Intestine Prevents Dietinduced Obesity in rats.Gastroenterology. 2018; 155(2): 501-513.

33. Lingzhi Li,Liang Ma,Ping Fu.Gut microbiota-derived short-chain fatty acids and kidney diseases.Drug Design, Development and Therapy 2017:11 3531-3542 


\section{Tables}

\section{Table 1}

High glucose increased blood IL-6 and TNF-a $(\mathrm{pg} / \mathrm{ml})$

\begin{tabular}{|lll|}
\hline & IL-6 & TNF-a \\
\hline OHG group & $122+11.16^{\square}$ & $295.28+36.95^{\square}$ \\
\hline HGI group & $127.8+16.42^{\square}$ & $300.28+30.1^{\square}$ \\
\hline Control group & $89.49+18.24$ & $245.85+34.46$ \\
\hline
\end{tabular}

$\bigotimes, P<0.01$ compared with the control group.

Compared to control group,blood IL-6 and TNF- $a$ of rats increased obviously after 2 weeks of oral high glucose and high glucose infusion.

\section{Figures}

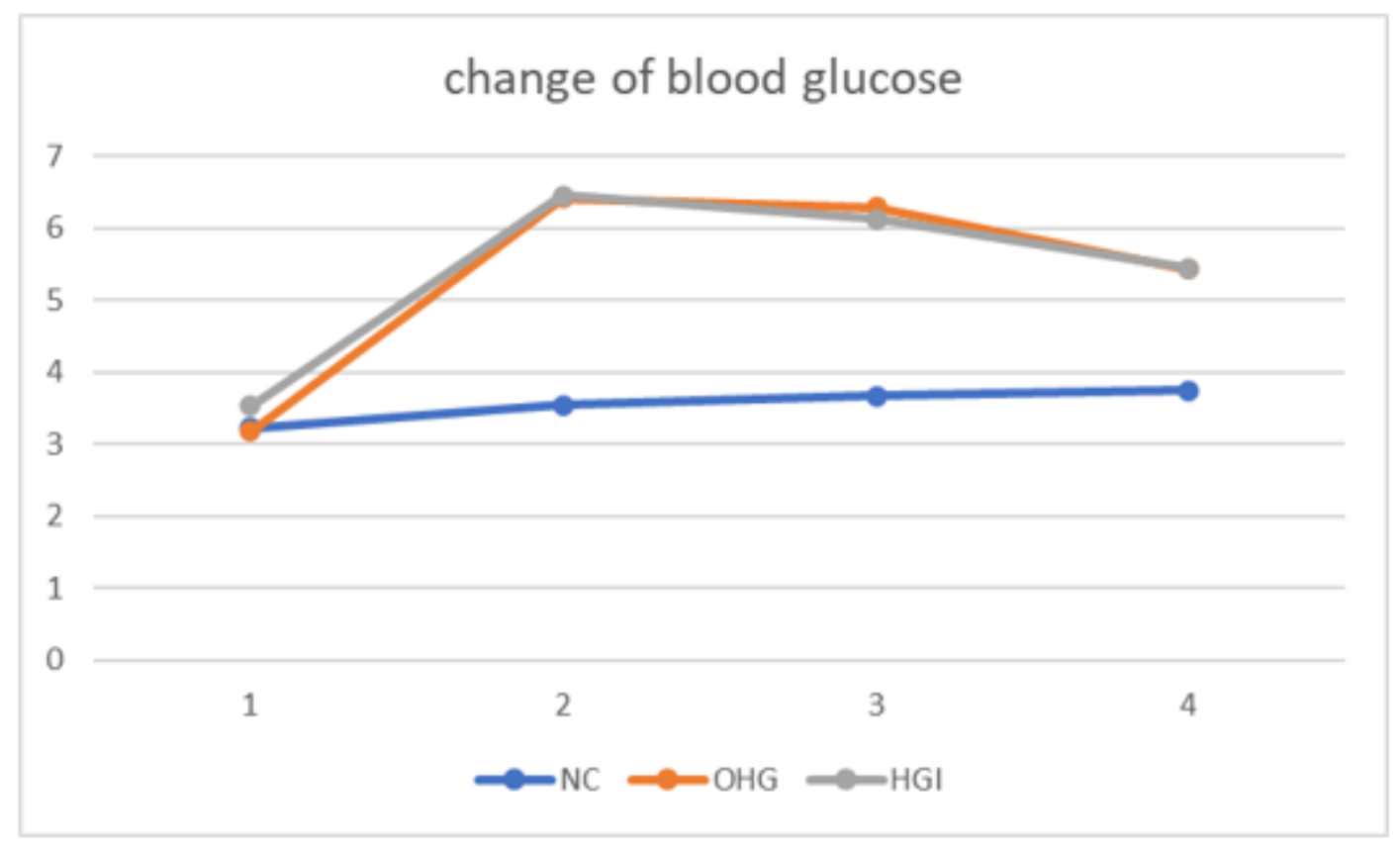

\section{Figure 1}

Change of blood glucose Blood glucose of rats in oral high glucose intake(OHG) group and high glucose infusion( $\mathrm{HGl}$ ) group was obviously higher than control group(NC) till 14 days after ceasing of glucose taking.There is no deference between oral high glucose intake and high glucose infusion 


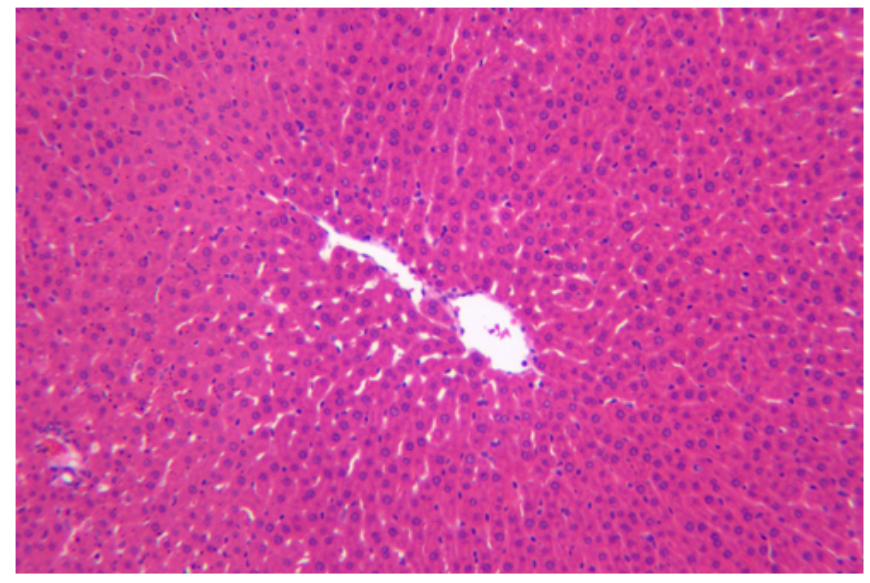

Figure 2A

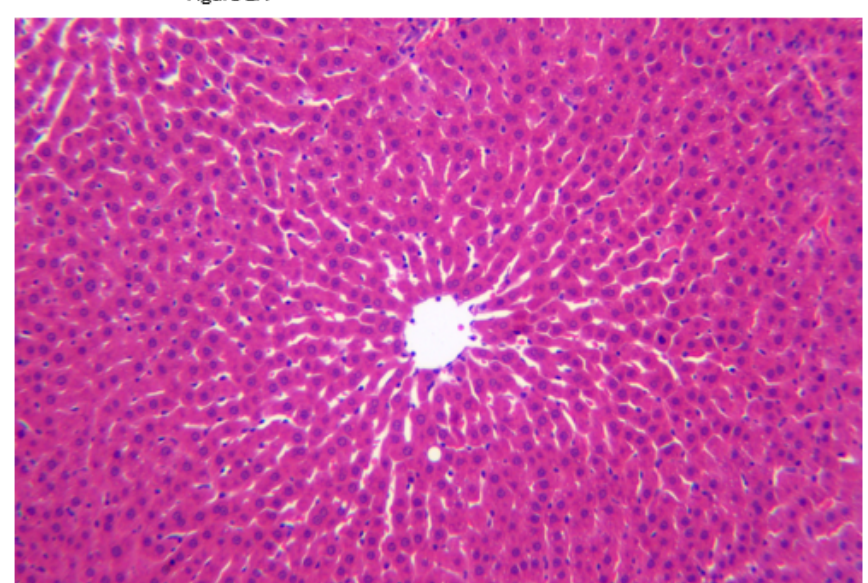

Figure $2 \mathrm{~B}$

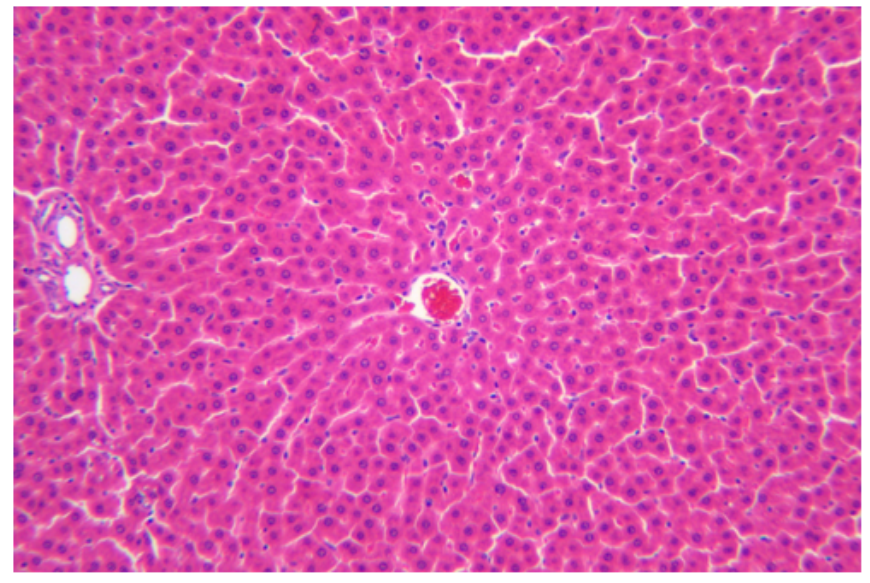

Figure 2C

\section{Figure 2}

Damage of glucose on liver A. liver of control group. X200 B. liver of oral high glucose group. X200 C. liver of high glucose infusion group. X200 Both oral high glucose and high glucose infusion induced obvious steatosis in rats liver. 


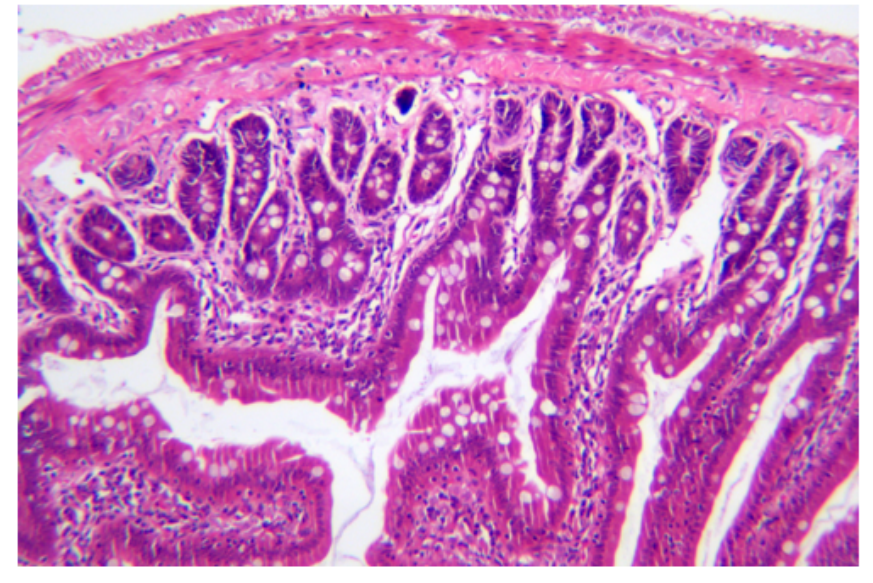

Figure $3 \mathrm{~A}$

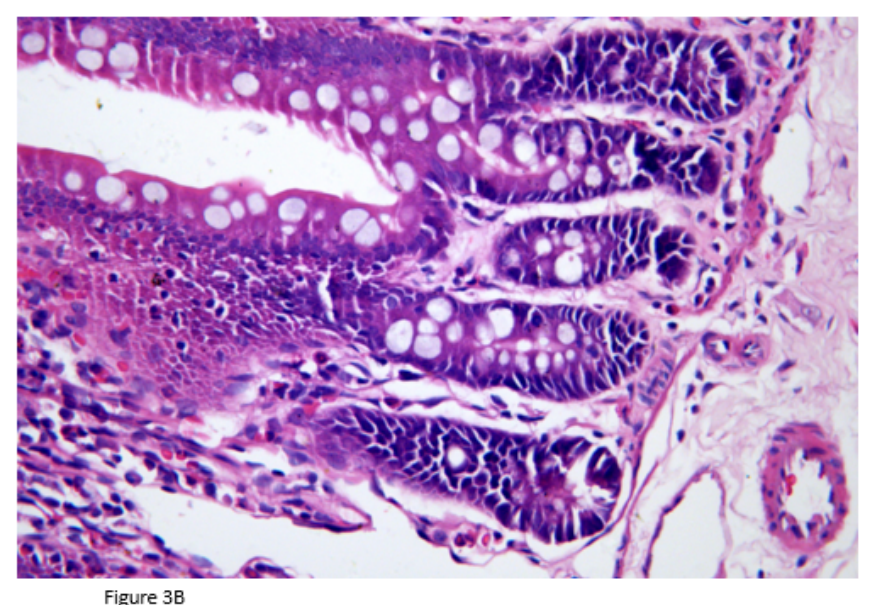

Figure 3B

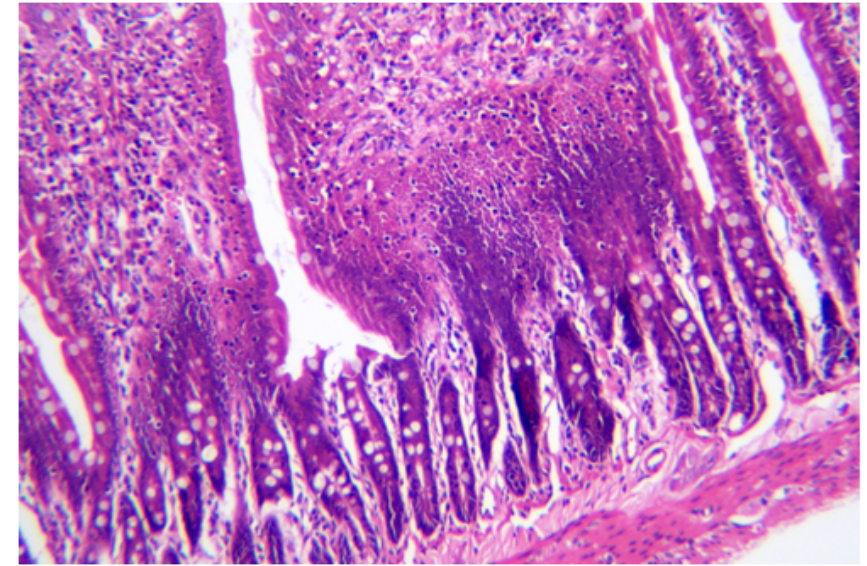

Figure $3 \mathrm{C}$

\section{Figure 3}

Damage of high glucose on intestinal mucosa A. Intestinal mucosa of control group. X200 B. Intestinal mucosa of oral high glucose group. X200 C. Intestinal mucosa of high glucose infusion group. X200 Oral high glucose led to swelling and necrosis of individual intestinal villi.Some epithelial cells disappeared.High glucose infusion led to necrosis and disappearence of cells in the upper layer of intestinal mucosa. The lesion was confined to the mucosa. 


\section{Weighted UniFrac Heatmap}

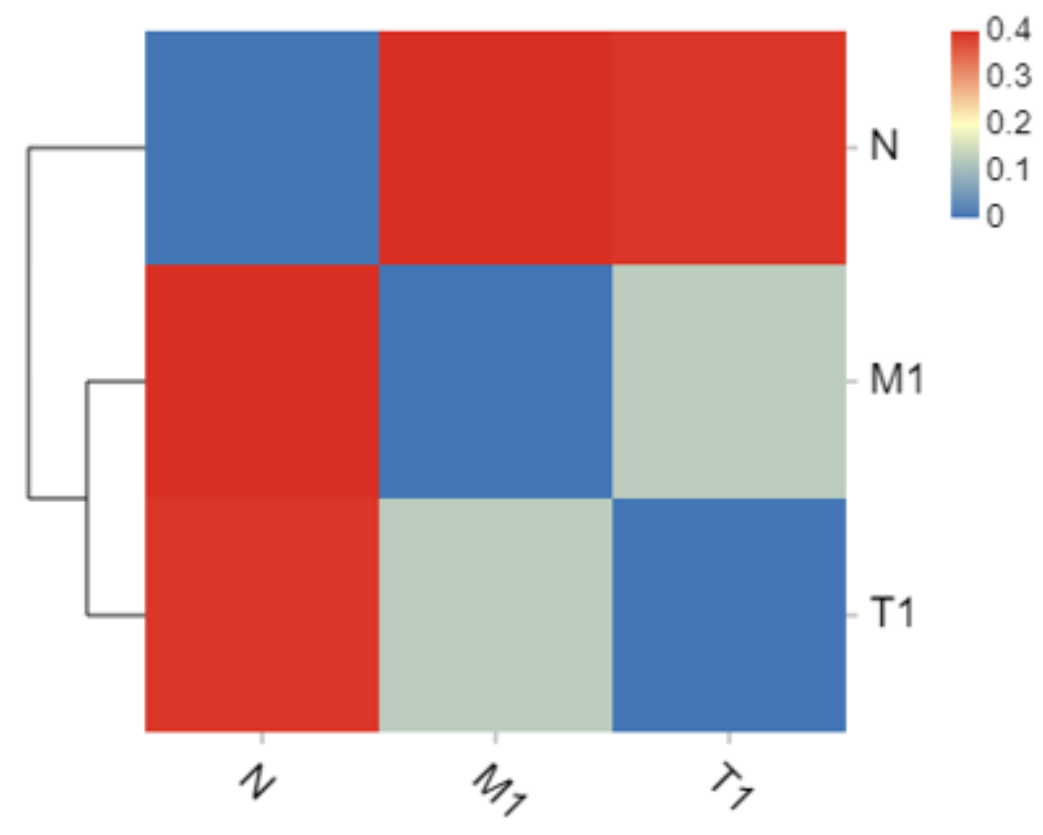

\section{Figure 4}

Gut microbiota change after high glucose intake Weighted UniFrac Heatmap of gut microbiota in SD rats.N,control group.M1,oral high glucose group.T1,high glucose infusion group.

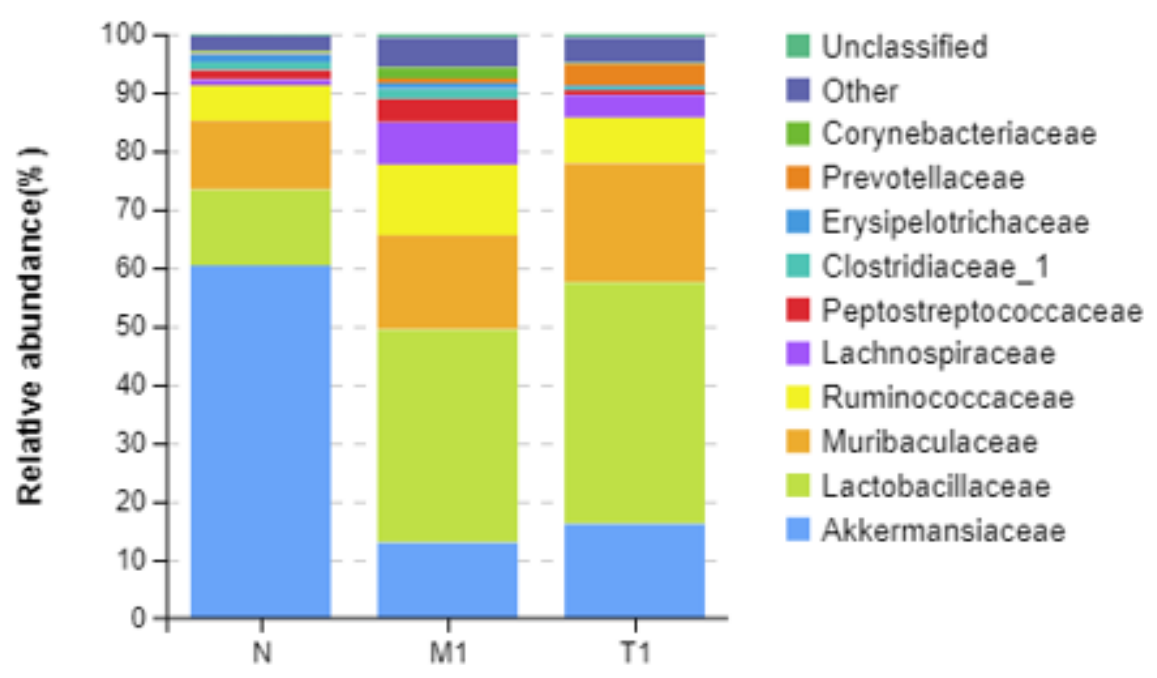

Figure 5

Gut microbiota change after high glucose intake Relative abundance of gut microbiota in SD rats. $\mathrm{N}$,control group. M1,oral high glucose group. T1, high glucose infusion group. 


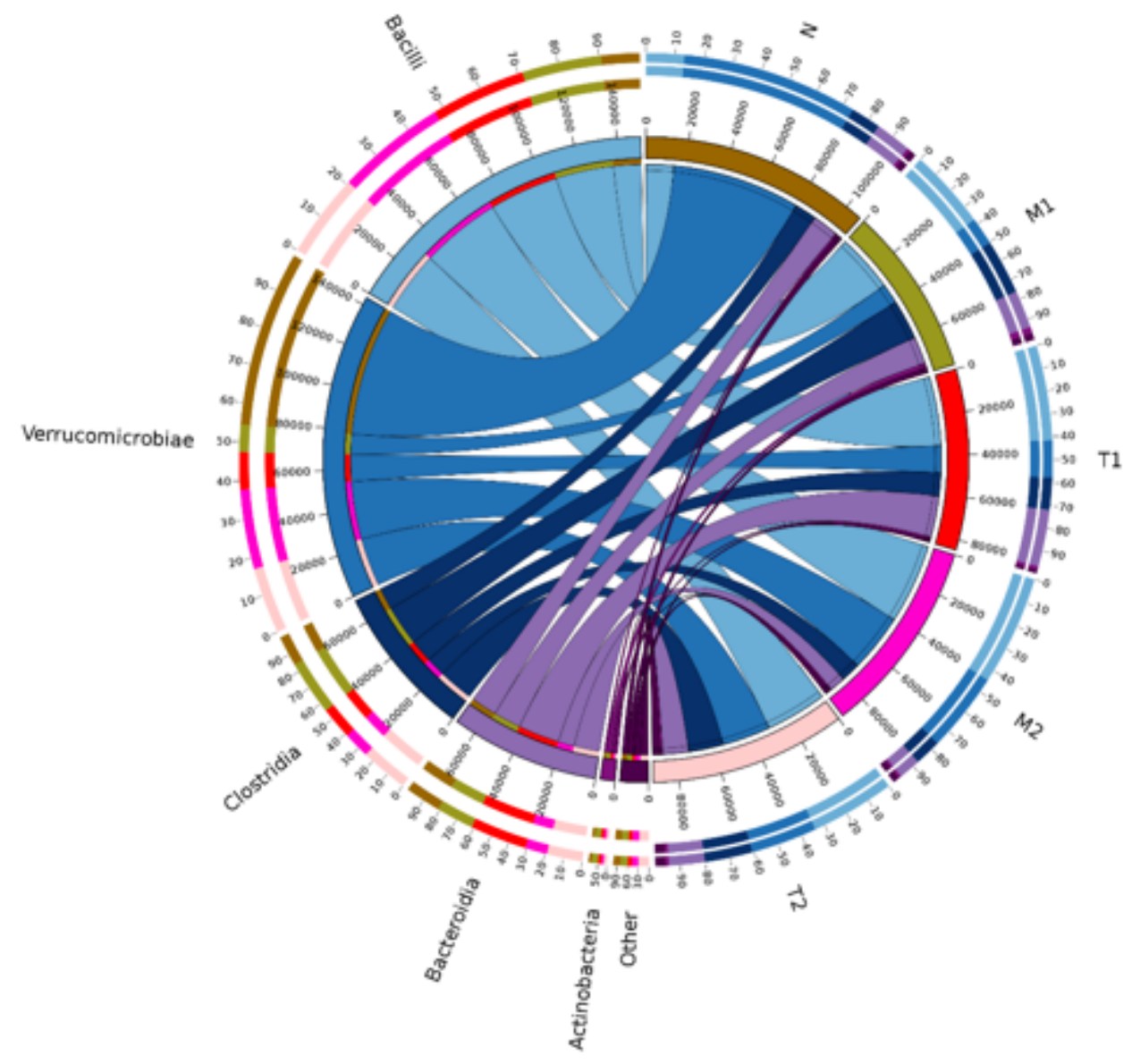

Figure 6

Circos of gut microbiota Circos of gut microbiota in SD rats. N,control groups. M1,2,oral high glucose groups. T1,2,high glucose infusion groups. 